\title{
Exploring diagnostic and therapeutic implications of endoscopic mucosal resection in EUS-staged $T 2$ esophageal adenocarcinoma
}

Authors

Annieke W. Gotink' ${ }^{1}$, Manon C. W. Spaander ${ }^{1}$, Michael Doukas ${ }^{2}$, Thjon J. Tang ${ }^{3}$, Paul Didden ${ }^{1}$, Bas P. L. Wijnhoven ${ }^{4}$, Marco J. Bruno ${ }^{1}$, Arjun D. Koch ${ }^{1}$

Institutions

1 Department of Gastroenterology and Hepatology, Erasmus MC, University Medical Center, Rotterdam, The Netherlands

2 Department of Pathology, Erasmus MC, University Medical Center, Rotterdam, The Netherlands

3 Department of Gastroenterology and Hepatology, IJsselland Hospital, Capelle aan den IJssel, The Netherlands

4 Department of Surgery, Erasmus MC, University Medical Center, Rotterdam, The Netherlands

submitted 7.11.2016

accepted after revision 8.5.2017

Bibliography

DOI https://doi.org/10.1055/s-0043-112492

Published online: 21.6.2017 | Endoscopy 2017; 49: 941-948

(c) Georg Thieme Verlag KG Stuttgart · New York

ISSN 0013-726X

Corresponding author

Arjun D. Koch, MD PhD, Department of Gastroenterology and Hepatology, Erasmus MC, University Medical Center Rotterdam, P.O. Box 2040, 3000 CA Rotterdam, The Netherlands

a.d.koch@erasmusmc.nl

Scan this QR-Code for the author's interview.

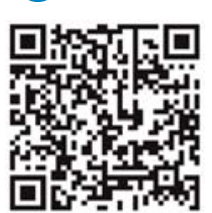

\section{ABSTRACT}

Background and study aims Treatment strategies for clinical (c)T2N0M0 esophageal adenocarcinoma (EAC) are subject to debate owing to the relative inaccuracy of tumor staging by endoscopic ultrasound (EUS), with profound implications in overstaged patients. We aimed to evaluate the final histological diagnosis of patients initially staged as having a cT2 tumor by EUS, and to assess the value of endoscopic reassessment by an interventional endoscopist, followed by an endoscopic resection when deemed feasible. Patients and methods Two distinct cohorts of patients with cT2 EAC as determined by EUS were included: a retrospective surgical cohort of patients treated by primary esophagectomy, and a prospective cohort of patients who underwent an endoscopic reassessment by an interventional endoscopist. The main outcome measure was the final pathological (p)T stage.

Results We identified 134 patients with stage T2 EAC from the surgical cohort. In 72 patients treated by primary esophagectomy, 32/72 (44\%) were downstaged to a pT1 tumor. In $12 / 72(17 \%)$, the surgical resection specimen showed tumor characteristics that fulfilled the current criteria for a curative endoscopic resection. In 13 prospectively identified patients with CT2N0M0 EAC, an expert endoscopic reassessment was done. In 11/13 (85\%) the lesion appeared endoscopically resectable and a complete endoscopic resection was performed. Histology revealed a pT1 tumor in all 11 patients, with 5/13 (38\%) fulfilling current criteria for a curative endoscopic resection.

Conclusions In this study, $44 \%$ of cT2 EACs were in fact PT1 tumors. Curative treatment by endoscopic resection was achieved in more than a third of these cases. To avoid an unnecessary esophagectomy, an endoscopic reassessment by an interventional endoscopist is recommended for all patients with cT2NOMO EAC.

\section{Introduction}

The incidence of esophageal adenocarcinoma (EAC) has increased rapidly over the last three decades [1,2]. Although therapeutic protocols for early- and late-stage esophageal tumors have been well defined, treatment strategies for clinicalstage T2N0M0 tumors (cT2N0M0) are subject to debate owing to the relative inaccuracy of endoscopic ultrasound (EUS) staging, with profound implications in overstaged patients [3-6]. A minimally invasive endoscopic resection is recommended for cT1 EACs. Only when the endoscopic resection specimen shows histological characteristics associated with an increased risk of lymph node metastasis (LNM) will a surgical resection follow [7-9]. For locally advanced tumors (cT2-4), however, neoad- 
juvant radiotherapy and/or chemotherapy followed by surgical resection is recommended [10].

Treatment decisions are based on the clinical tumor stage, for which imaging techniques such as EUS, computed tomography (CT), ultrasound of the neck, and positron emission tomography (PET) are used $[11,12]$. EUS is accurate in staging T3 and T4 tumors and locoregional LNM, but the accuracy is lower when it comes to differentiating $\mathrm{T} 2$ from $\mathrm{T} 1$ tumors, with a sensitivity of $43 \%-55 \%$ and a specificity of $80 \%-85 \%[13,14]$. In T2 tumors that are inaccurately staged by EUS, the final pathology reveals a pathological (p)T0-1 stage in $21 \%-63 \%$ and a PT3 - 4 N0 or PT any N1 - N3 in 19\%-50\% [5, 6,15].

Current debates on the optimal treatment approach for cT2N0M0 EACs compare primary surgical resection to neoadjuvant chemoradiotherapy (nCRTx) followed by surgery. To date, no studies have investigated whether an endoscopic reassessment followed by endoscopic resection for potentially overstaged cT2 tumors is of added value to patient management. Therefore, the aim of this study was twofold: first, to evaluate the final histological diagnosis of patients initially diagnosed with a CT2 EAC on the basis of EUS evaluation; and second, to assess the value of an endoscopic reassessment by an interventional endoscopist, followed by endoscopic resection if deemed possible. Because of the potential risk of overstaged cT2 tumors, in recent years we have adopted a policy of being suspicious of EACs initially staged as being cT2, with a low threshold for endoscopic reassessment by an interventional endoscopist.

\section{Patients and methods}

\section{Patients}

Two distinct cohorts of patients with a CT2 EAC as determined by EUS were included in this study: a retrospective series of patients who underwent surgical resection, and a prospective cohort of patients who underwent an endoscopic reassessment followed by endoscopic resection when deemed possible. The study was approved by the institutional review boards of the participating centers.

\section{Surgical cohort}

Patients were identified from the Erasmus MC, University Medical Center, Rotterdam, The Netherlands surgical database. A specialized data manager prospectively registered data on patient demographics, preoperative tumor staging, postoperative pathological staging, and follow-up. All patients with a cT2 EAC as determined by EUS who underwent surgical tumor resection without preoperative chemoradiotherapy between January 1990 and October 2014 were included in this study.

Patients were excluded if their tumor was a gastric tumor, a squamous cell carcinoma, when clinical staging by EUS was not possible because of obstruction, or when an (attempt at) endoscopic resection had taken place prior to EUS staging.

\section{Endoscopic reassessment cohort}

Patients were included at the Erasmus MC, University Medical Center, Rotterdam and at the IJsselland Hospital, Capelle aan den Ijssel. Both centers in The Netherlands are specialized in the endoscopic treatment of early esophageal tumors, with a low threshold for endoscopic reassessment of cT2N0M0 tumors as part of routine medical care. Between January 2011 and November 2014, all cT2 patients in whom an endoscopic reassessment was done were registered prospectively.

\section{Pre-operative tumor staging}

After an initial diagnosis of esophageal cancer by standard endoscopy, a conventional radial EUS, CT scan of the thorax and upper abdomen, and ultrasound of the neck was performed to assess the TNM stage. A PET CT was not part of routine tumor staging, but was performed in some instances. As the Erasmus MC, University Medical Center Rotterdam and the IJsselland Hospital are tertiary referral centers, initial staging was often done elsewhere. When the initial EUS had reported possible lymph node involvement, the examination was repeated with fine needle aspiration if this would have altered patient's management.

Tumors were considered cT2 tumors based on the findings of EUS. In some instances, the EUS was reported as being unable to adequately discriminate between a cT1 or cT2 tumor (referred to as a cT1 - 2 tumor in the EUS report). Such patients were considered to have a cT2 tumor and were referred for a surgical resection. Accordingly, they were included in our study as cT2 tumors.

\section{Endoscopic reassessment and resection}

Endoscopic reassessment for potentially overstaged patients was limited to patients who had no evidence of lymph node or distant metastases, because the goal was to prevent an unnecessary esophagectomy in these overstaged patients. Patients were selected at multidisciplinary meetings or from the outpatient clinic of the department of gastroenterology and hepatology. The criteria for inclusion were: cT2 or cT1-2 staged by EUS at any center; no evidence of lymph node or distant metastases on imaging and/or biopsy; and the lesion not having been previously assessed by an expert interventional endoscopist.

During endoscopic reassessment by an experienced interventional endoscopist, the endoscopic resectability of the lesion was assessed. Using high resolution endoscopy, the lesion was assessed for invasive features, such as the non-lifting sign, not moving freely with peristalsis, and the presence of deep ulceration or stricture. In the absence of invasive features, the lesion was deemed a cT1 tumor and an endoscopic resection was subsequently attempted. Depending on the lesion characteristics and location, this was done by either endoscopic mucosal resection (EMR) or endoscopic submucosal dissection (ESD). Prior to endoscopic resection, the lesion was delineated with coagulation markings, after which the delineated area was completely resected to ensure complete lateral resection of the lesion.

\section{Histological evaluation}

The pathology reports of all surgical and endoscopic resection specimens were reviewed for tumor depth infiltration, tumor differentiation (classified as good [G1], moderate [G2], and 
poorly differentiated/undifferentiated [G3/G4]), the presence of lymphovascular invasion (LVI), and involvement of the resection margins (R0/R1), according to the latest World Health Organization (WHO) classification. For PT1 tumors, this was divided into either mucosal or submucosal tumors, with the submucosa further subdivided into sm $1(\leq 500 \mu \mathrm{m})$ or sm $2-3$ (according to the Paris Classification [16]). Where the pathology reports were incomplete or did not conform to the latest WHO classification, an expert gastrointestinal pathologist re-evaluated the histopathology.

Surgical and endoscopic resection specimens were assessed to determine whether they fulfilled the current pathological criteria for curative treatment by endoscopic resection, where the risk of LNM is negligible. These favorable criteria are: maximum depth of tumor infiltration is limited to the mucosa or upper $500 \mu \mathrm{m}$ of the submucosa (pT1m1-3sm1), in combination with good or moderate tumor differentiation (G1/G2) and no LVI (pT1m1-3sm1N0M0, G1/G2, no LVI) [7, 17-22]. Additionally, in endoscopic resection specimens, the vertical resection margins should not be involved (R0). Lateral resection margins of piecemeal resection specimens are nearly always $\mathrm{R} 1$. The determination of whether lateral margins are tumor free is done endoscopically and not pathologically. If, after endoscopic resection, the pathological criteria were met and the vertical resection margins were not involved (R0), indicating a curative resection, no subsequent esophagectomy was carried out.

\section{Study outcomes}

In the surgical cohort, the primary outcome was assessment of how many patients were overstaged based on the PT stage. The secondary outcome parameter was assessment of how many patients fulfilled the pathological criteria for a curative endoscopic resection.

In the endoscopic reassessment cohort, the primary outcome was assessment of how many patients were overstaged after endoscopic reassessment of the lesion. The secondary outcome parameter was assessment of how many patients fulfilled the pathological criteria for a curative endoscopic resection.

\section{Statistical methods}

Statistical analysis was performed using the statistical software IBM SPSS Statistics 21. Data were analyzed descriptively using means and standard deviations for normally distributed data; medians and ranges for skewed data. In the endoscopic reassessment cohort, the median follow-up time was calculated using the time between the endoscopic tumor resection and the date of the most recent clinical, endoscopic, or radiological investigation.

\section{Results}

\section{Surgical cohort}

Patients

From a total of 1790 patients with an EAC, 134 patients (7\%) had a CT2 tumor as determined by EUS. From those 134 patients with a cT2 EAC, 72 patients (54\%) were treated by pri-
- Table 1 Baseline characteristics of the 72 patients with a cT2 esophageal adenocarcinoma as determined by endoscopic ultrasound who underwent surgical tumor resection without preoperative chemoradiotherapy.

Sex, male, $\mathrm{n}(\%)$ $61(85 \%)$

Age at diagnosis, median (range), years $64(39-81)$

Reported endoscopic resectability, $\mathrm{n}$ (\%)

- Not reported 69 (96\%)

- Resectable 0

- Not resectable $3(4 \%)$

Clinical tumor staging, $\mathrm{n}(\%)$

- cT1-2N0M0 $4(6 \%)$

- $\mathrm{CT} 1-2 \mathrm{~N} 1 \mathrm{M} 0$ 1 (1\%)

- cT2NOMO $51(71 \%)$

- cT2N1M0 $16(22 \%)$

Type of esophagectomy

- Transhiatal $58(81 \%)$

- Transthoracic $12(17 \%)$

Gastrectomy $2(3 \%)$

Surgery-related mortality $3(4 \%)$

mary esophagectomy; 36 patients (27\%) were treated by neoadjuvant therapy followed by esophagectomy; and in 26 patients (19\%), no esophagectomy was performed. In this last group, patients received definitive chemoradiotherapy, palliative treatment, or no additional therapy because of metastatic disease, patient comorbidity, or patient preference. The characteristics of the 72 patients with a CT2 EAC who were treated by primary esophagectomy are shown in $>$ Table 1 .

\section{Pathological tumor stage}

Of the 72 patients with a CT2 EAC who underwent a primary esophagectomy, 32 patients (44\%) had a pT1 stage; 17 patients $(24 \%)$ had a pT2 stage; and 23 patients (32\%) had a pT3 stage ( $\triangleright$ Fig. $\mathbf{1})$. In 12 patients (17\%), the surgical resection specimen showed tumor characteristics that fulfilled current criteria for curative treatment by endoscopic resection and no lymph metastases were found (pT1m1-3sm1N0M0, G1/G2, no LVI), with 10 patients having mucosal tumors and two having an sm 1 tumor with low risk other histological characteristics. In five patients with a submucosal tumor, histopathology was not available for re-evaluation; these patients were therefore categorized as not fulfilling the current criteria for an endoscopic resection.

\section{Endoscopic reassessment cohort}

\section{Patients}

In 13 prospectively identified patients with cT2NOMO EAC, an endoscopic reassessment was carried out to evaluate endo- 


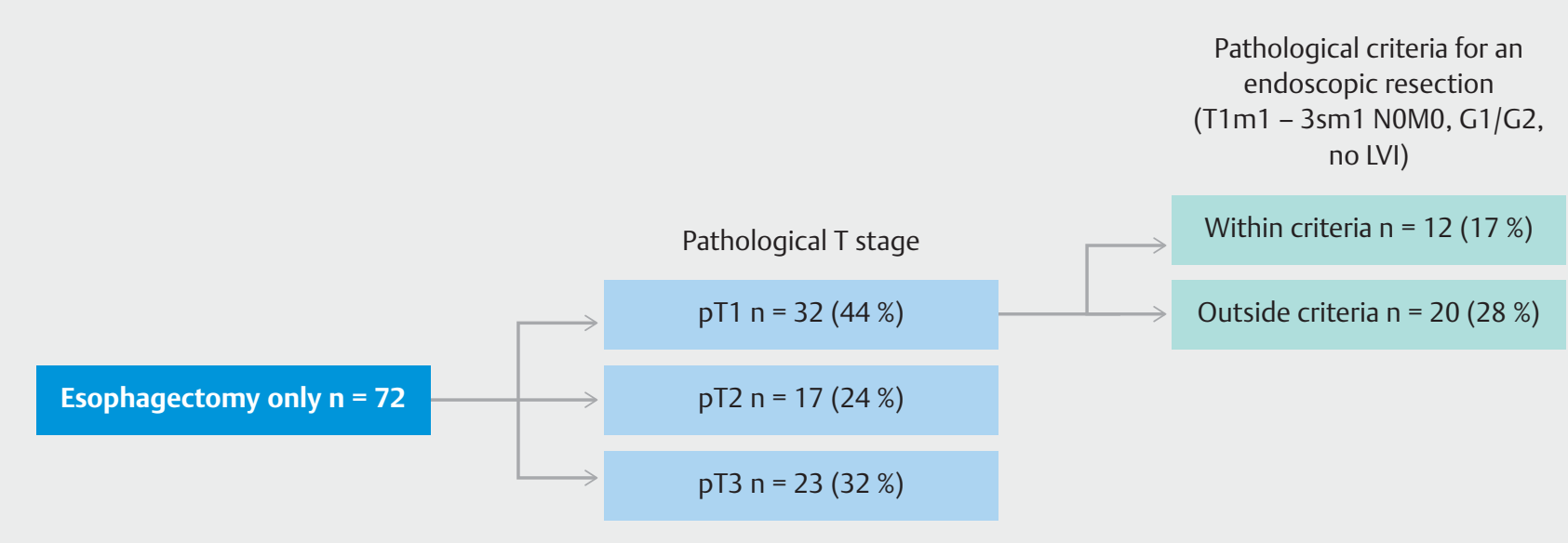

- Fig. 1 Pathological tumor stage of cT2 patients who underwent surgical tumor resection without preoperative chemoradiotherapy.

- Table 2 Baseline characteristics of the 13 patients with a cT2 esophageal adenocarcinoma as determined by endoscopic ultrasound who underwent endoscopic reassessment.

\begin{tabular}{|l|c|}
\hline Gender, male, $\mathrm{n}(\%)$ & $13(100 \%)$ \\
\hline Age at diagnosis, median (range), years & $66(50-81)$ \\
\hline Reported endoscopic resectability, $\mathrm{n}(\%)$ & \\
\hline - Not reported & 0 \\
\hline - Resectable & $11(85 \%)$ \\
\hline - Not resectable & $2(15 \%)$ \\
\hline Clinical tumor staging $\mathrm{n}(\%)$ & \\
\hline - cT1-2N0M0 & $3(23 \%)$ \\
\hline - cT2NOMO & $10(77 \%)$ \\
\hline
\end{tabular}

scopic resectability. The characteristics of these patients are shown in $>$ Table 2.

In 11 patients (85\%), the lesion was endoscopically staged as a T1 tumor because of the absence of invasive features, and an endoscopic resection was subsequently attempted ( $\mathbf{F i g . 2}$ ). In all 11 patients, the endoscopic resection was successfully performed by EMR. Endoscopic characteristics of the lesions are described in > Table 3. In 10/11 patients, the lesions were described as either a pedunculated, sessile, or elevated.

In two patients (15\%), the T stage was considered to correspond with the EUS stage, as invasive features were seen during endoscopic reassessment. Both of these patients were referred for chemoradiotherapy followed by esophagectomy.

\section{Pathological tumor stage}

The pathological characteristics of the 11 patients with a CT2 EAC who underwent endoscopic reassessment followed by endoscopic resection are shown in $>$ Table 3 . In five patients (38\%), the endoscopic resection specimen showed tumor characteristics that fulfilled the current criteria for curative treatment by endoscopic resection. In a median follow-up of 28.3
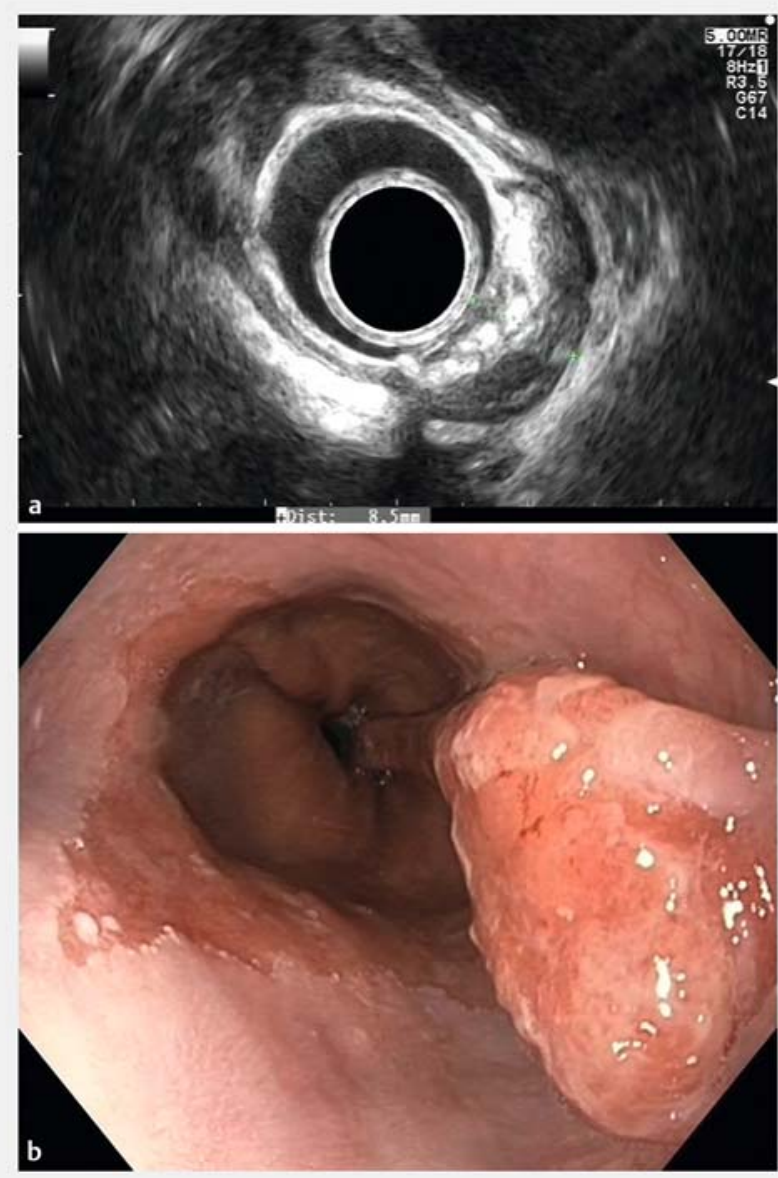

- Fig. 2 Example of a cT2 esophageal adenocarcinoma (EAC), staged by endoscopic ultrasound, which was downstaged to a CT1 tumor after endoscopic reassessment by an interventional endoscopist: a Endoscopic ultrasound appearance indicating a cT2 EAC. b Endoscopic reassessment of a lesion without invasive features. Final histology after endoscopic resection was pT1sm1, G2, no LVI. 


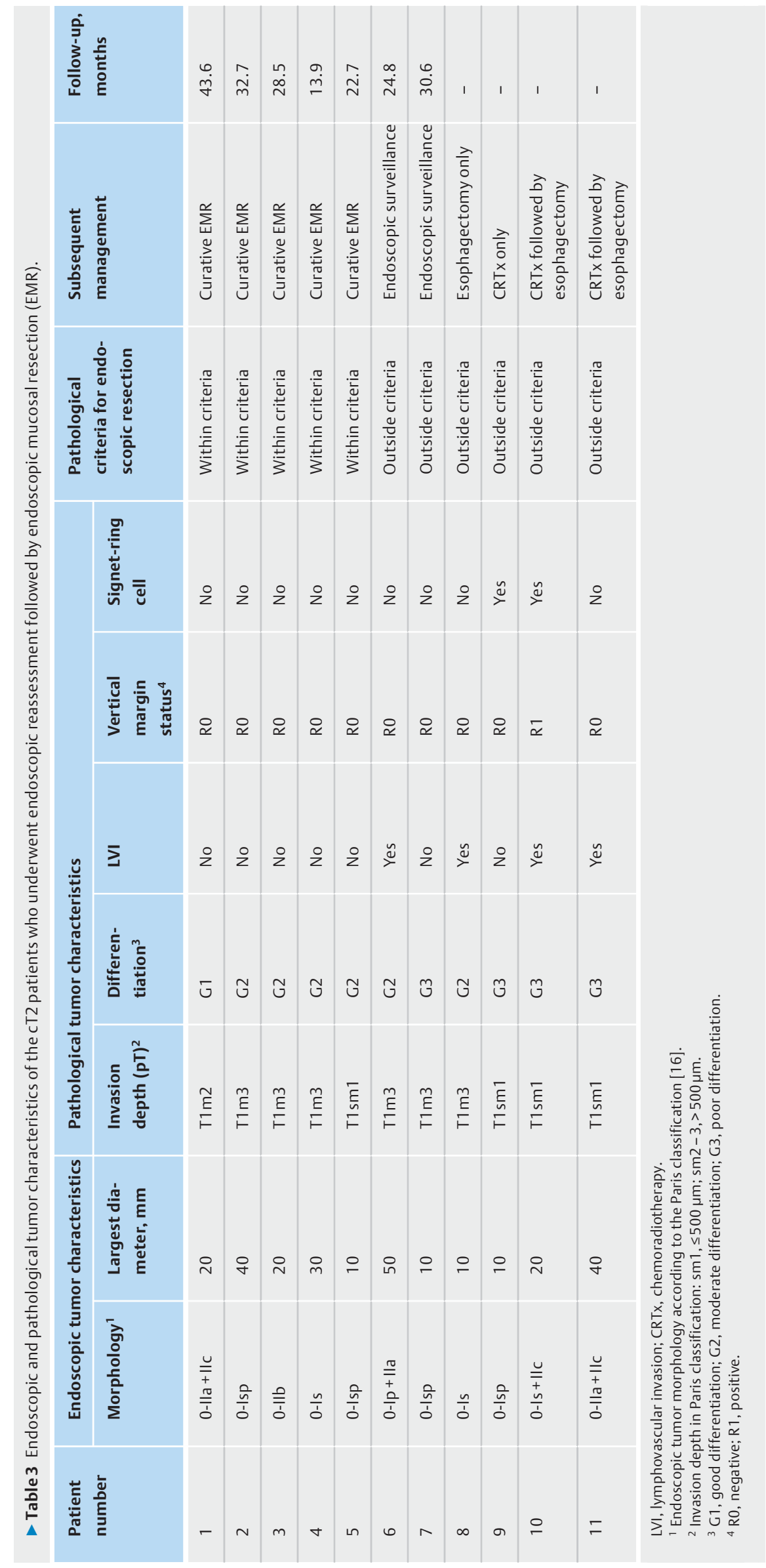


months (range 13.9-43.6 months), there were no signs of LNM or distant metastatic disease.

Of the six patients ( $46 \%$ ) that fell outside these criteria, one patient was inoperable and was treated by chemoradiotherapy; two were referred for chemoradiotherapy followed by esophagectomy, with the decision for neoadjuvant therapy based on the increased risk of LNM associated with the poor pathology; and one underwent a primary esophagectomy, where a residual mucosal tumor (M2) and no evidence of LNM was found in 18 resected lymph nodes. The last two patients have remained under close endoscopic surveillance; with follow-up of 24.8 and 30.6 months, there were no signs of LNM or distant metastases in these two patients.

\section{Adverse events associated with endoscopic resection}

In 2/11 patients who underwent an EMR procedure, an adverse event occurred. One patient had a minor perforation that was successfully treated by clips (1-day hospitalization); the other patient had a post-EMR stricture that required multiple dilations. In both patients, the endoscopic resection specimen fulfilled the criteria for a curative treatment by endoscopic resection.

\section{No endoscopic reassessment}

A retrospective review of the surgical cohort between January 2011 and October 2014 showed that 16 patients were diagnosed with a cT2 EAC but did not undergo an endoscopic reassessment. In nine patients, there was a suspicion of LNM and/or distant metastasis; in one patient, it was documented that endoscopic reassessment would not be of added value because biopsies showed poor tumor differentiation; in six patients, the reasons were unknown. The 16 patients underwent different treatment strategies: 11 were treated by chemoradiotherapy followed by esophagectomy; in two patients, no esophagectomy was performed; and three patients were treated by primary surgery, with the final histology revealing a submucosal tumor in two patients.

\section{Discussion}

This study demonstrates that EUS has a poor accuracy for staging CT2 EAC, considering 44\% of cT2 EACs were downstaged to pT1 tumors after histological examination of the esophagectomy specimens. We have established that, after endoscopic reassessment by an interventional expert, curative treatment by endoscopic resection is possible in up to $38 \%$ of cT2N0M0 patients, thereby avoiding the need for chemoradiotherapy followed by esophagectomy.

In the last three decades, the management of esophageal cancer has evolved significantly [3]. In the past, primary surgical resection was the only treatment for esophageal cancer or high grade dysplasia. At present, minimally invasive endoscopic resection is a recommended treatment modality for cT1 tumors, after which additional surgery is required only for those patients with poor histological characteristics associated with an increased risk of LNM [7-9]. The use of nCRTx followed by surgery has been widely accepted for locally advanced (cT24) tumors $[3,10]$. These treatment modalities have demonstrated improved survival compared with surgery alone and have a significant impact on quality of life in patients with esophageal cancer $[2,7,10]$.

Endoscopic resection was initially accepted only for mucosal EAC because of the negligible risk of LNM in these superficial tumors. However, numerous new studies have shown that when the tumor extends into the upper $500 \mu \mathrm{m}$ of the submu$\operatorname{cosa}(\operatorname{sm} 1)$, in combination with a good or moderate tumor differentiation (G1/G2) and no LVI, the risk of LNM remains negligible [18-22]. Accordingly, new guidelines have cautiously recommended minimally invasive endoscopic resection for these low-risk sm 1 tumors $[7,17]$, and many centers have already implemented this strategy with stringent follow-up. For this reason, in our study, low-risk sm 1 tumors were also considered to fulfill the current pathological criteria for curative treatment by endoscopic resection. In support of these new recommendations, no LNM were found in the two patients with low-risk sm1 tumors in our surgical cohort; in our endoscopic reassessment cohort with one low-risk sm 1 patient, there were no signs of LNM or distant metastatic disease during a follow-up of 22.7 months.

Accurate tumor staging is crucial in establishing the appropriate choice of treatment. EUS is superior to CT and PET in delineating the esophageal wall layers and determining the appropriate T stage $[5,23,24]$, but it has poor accuracy when differentiating T2 from T1 tumors, with a sensitivity of $43 \%-55 \%$ and specificity of $80 \%-85 \%[13,14]$. In our surgical cohort, 44 $\%$ of cT2 EACs could be downstaged to PT1 tumors based on the final pathology report, which is a similar finding to previous studies reporting downstaging in $21 \%-63 \%$ of cases $[3,6,15$, $25]$. Moreover, in $17 \%$ of patients, histopathology even fulfilled the current criteria for curative endoscopic resection, and potentially a surgical resection could have been avoided if an endoscopic resection had been attempted.

In the clinical setting, imaging techniques for tumor staging are performed after initial endoscopic assessment of a lesion when an advanced tumor $(T>1)$ is suspected. In the absence of invasive features (T1 lesion), an endoscopic resection is advised and guidelines advocate referral to a high-volume tertiary center because of the expertise and skills required for this type of intervention [7]. In those with a CT1 tumor after further tumor staging by EUS, the patient will nevertheless be referred for an endoscopic resection. In those with a cT2 tumor, however, guidelines recommend treatment by nCRTx followed by surgical resection, although it is known that EUS poorly differentiates between T2 and T1 tumors. For the general endoscopist, competence in the endoscopic recognition of invasive features is therefore a crucial step in deciding between further tumor staging by imaging or referral for an endoscopic resection.

Many studies have investigated new imaging techniques to enhance the detection of dysplasia and neoplasia [17]. These studies, however, scarcely describe how to determine whether a lesion is endoscopically resectable once detected. In our endoscopic reassessment cohort, most lesions were described 
as being pedunculated, sessile, or elevated. A possible explanation for the poor recognition might be that these lesions were not regarded as suitable for endoscopic resection because they appeared large and were presumed to carry a risk of LNM. Although some studies identify tumor size and morphology as predictors for LNM in submucosal EAC $[26,27]$, it should be noted that histopathological assessment of an endoscopic resection specimen is the most accurate staging technique for early Barrett's neoplasia, providing a histological profile to help predict the risk of LNM [7].

Guidelines advise an endoscopic resection when the lesion involves less than one-third of the circumference of the esophageal wall and when the maximum diameter is less than or equal to $2 \mathrm{~cm}[28,29]$. Technically, larger lesions can be effectively resected, but complications such as stricture, perforation, or bleeding will occur more often [30]. Piecemeal resection by EMR and en bloc resection by ESD are both highly effective for the endoscopic resection of early Barrett's neoplasia in terms of neoplasia remission and recurrence. ESD is more timeconsuming and technically difficult, but does allow for the resection of larger lesions [31,32]. In our study, lesions were resected by EMR in all 11 patients, and two of these patients had a complication due to the procedure; both were mild and treated by endoscopic means. This does not compare to the reported morbidity and mortality rates of $20 \%-80 \%$ and $0 \%-22 \%$, respectively, associated with a surgical resection [33].

In a trial comparing high resolution endoscopy to EUS using a high resolution miniprobe, May et al. [34] demonstrated that endoscopic staging has a high sensitivity of $82.9 \%$ and an accuracy of $83.4 \%$, which was slightly superior to, but not significantly different from, that of EUS staging. In this trial, only experienced interventional endoscopists were involved in tumor staging. In our endoscopic reassessment cohort, endoscopic tumor staging by an experienced interventional endoscopist was superior to EUS staging using a conventional radial echoendoscope, as $85 \%$ of cT2 lesions could be downstaged to cT1 lesions.

Most endoscopists traditionally are not trained in assessing the endoscopic resectability of an EUS-staged T2 EAC, and therefore a potentially curative and minimally invasive treatment option such as endoscopic resection is not considered further. The EUS verdict is often regarded as an "absolute truth" and these patients are therefore routinely referred for nCRTx followed by surgery. Because of the low threshold for endoscopic reassessment, in our prospective cohort, $38 \%$ of patients were curatively treated by endoscopic resection. This suggests that all patients with an EAC staged as CT1 or CT2 should be reassessed endoscopically at a tertiary referral center by an experienced interventional endoscopist.

The question may arise as to whether the use of EUS is obsolete in assessing tumor infiltration depth [35], because it could be argued that a lesion is either endoscopically resectable $(T<$ 2 ) or not $(T \geq 2)$, which clinically implies treatment by either endoscopic resection or by esophagectomy with or without (neo)adjuvant chemoradiation. This is an issue often debated, but to date there is no literature to support such a strategy. It may be a valid point for early tumors when there is adequate re- cognition of an endoscopically resectable lesion. However, we demonstrate that endoscopic staging is poor when performed by a general endoscopist. To date, EUS is the most accurate technique to determine the cT stage; EUS could, in essence, be used as a tool to distinguish who should be referred for an endoscopic reassessment by an experienced interventional endoscopist.

This study has several strengths and limitations. First, there is a selection bias in the endoscopic reassessment cohort as only a selection of all newly staged CT2 patients underwent an endoscopic reassessment. Only those that had undergone an endoscopic reassessment were prospectively registered. In our endoscopic reassessment cohort, $38 \%$ were curatively treated by endoscopic resection. However, when an estimate is made of all new CT2 patients based on both cohorts, at least $43 \%$ of patients were overstaged and $14 \%$ were curatively treated by endoscopic resection. These results are in line with the surgical cohort, where $44 \%$ had a PT 1 stage and $17 \%$ had tumor characteristics that fulfilled the current criteria for curative treatment by endoscopic resection.

A second limitation is that information bias may have occurred because data were retrospectively collected. However, determining the true pathological tumor stage will only be possible in retrospective surgical cohorts because, currently in most centers, all CT2 patients will be treated by nCRTx prior to surgery, with tumor downstaging as a consequence.

A third limitation is that we included a relatively small number of patients who underwent an endoscopic reassessment. Ideally, an adequately powered study should be performed to assess whether this approach statistically reduces the number of surgical resections in CT2 patients. Although the small sample is a limitation, it should be noted that the prevalence of patients with a CT2 EAC is low [15]. This is also demonstrated in our surgical cohort with 134 cT2 patients in a 24-year time period. Regardless of the low prevalence, the incidence of EAC is rapidly increasing and the clinical impact on these patients is very significant.

A fourth limitation is that the surgical cohort extends over a significant period of time, when an evolution in endoscopic treatment modalities was taking place. In the surgical cohort, $17 \%$ of patients fulfilled the criteria for curative endoscopic resection; however, EMR was only introduced at the Erasmus University Medical Center in 1999.

A final limitation is that we defined cT1 -2 tumors as CT2 tumors. These patients were referred for a surgical resection and were therefore included in our study. Clinically, the endoscopist performing the EUS should always define a tumor as a CT1 or a CT2 tumor and report the highest staging that was observed because the tumor either invades the muscularis propria or not. Poor sensitivity of EUS for CT2 EAC, however, supports the fact that it is not always easy to distinguish between these two. Such patients should therefore always be assessed by an expert endoscopist with a substantial amount of experience in staging and resection of neoplastic lesions.

In conclusion, an endoscopic reassessment by an experienced interventional endoscopist should be considered for all patients with a cT2NOMO EAC. This is the first study that de- 
monstrates that curative treatment by endoscopic resection is possible in up to $38 \%$ of patients, thereby avoiding the need for chemoradiotherapy followed by esophagectomy.

\section{Competing interests}

None

\section{References}

[1] Pohl H, Welch HG. The role of overdiagnosis and reclassification in the marked increase of esophageal adenocarcinoma incidence. J Natl Cancer Inst 2005; 97: 142 - 146

[2] Pennathur A, Gibson MK, Jobe BA et al. Oesophageal carcinoma. Lancet 2013; 381: $400-412$

[3] Hardacker T], Ceppa D, Okereke I et al. Treatment of clinical T2N0M0 esophageal cancer. Ann Surg Oncol 2014; 21: 3739-3743

[4] Speicher P], Ganapathi AM, Englum BR et al. Induction therapy does not improve survival for clinical stage T2N0 esophageal cancer. J Thorac Oncol 2014; 9: 1195 - 1201

[5] Vallbohmer D, Sisic L, Blank S et al. Clinically staged cT2 adenocarcinomas of the gastroesophageal junction: accuracy of staging and therapeutic consequences. Oncol Res Treat 2014; 37: 97 -104

[6] Crabtree TD, Kosinski AS, Puri V et al. Evaluation of the reliability of clinical staging of T2 N0 esophageal cancer: a review of the Society of Thoracic Surgeons database. Ann Thorac Surg 2013; 96: 382 - 390

[7] Fitzgerald RC, di Pietro M, Ragunath K et al. British Society of Gastroenterology guidelines on the diagnosis and management of Barrett's oesophagus. Gut 2014; 63: 7-42

[8] Spechler S], Sharma P, Souza RF et al. American Gastroenterological Association technical review on the management of Barrett's esophagus. Gastroenterology 2011; 140: e18-e52

[9] Evans JA, Early DS, Chandraskhara V et al. The role of endoscopy in the assessment and treatment of esophageal cancer. Gastrointestinal Endoscopy 2013; 77: $328-334$

[10] van Hagen P, Hulshof MCCM, van Lanschot JJB et al. Preoperative chemoradiotherapy for esophageal or junctional cancer. NEJM 2012; 366: $2074-2084$

[11] Khanna LG, Gress FG. Preoperative evaluation of oesophageal adenocarcinoma. Best Pract Res Clin Gastroenterol 2015; 29: 179-191

[12] Didden P, Spaander MCW, Wijnhoven BPL et al. Improving the quality of pretreatment staging in patients with esophageal carcinoma - a fast track study. Acta Oncologica 2012; 51: $362-367$

[13] O'Farrell N, Malik V, Donohoe C et al. Appraisal of Staging Endoscopic Ultrasonography in a Modern High-Volume Esophageal Program. World J Surg 2013; 37: $1666-1672$

[14] Pech O, Günter E, Dusemund F et al. Accuracy of endoscopic ultrasound in preoperative staging of esophageal cancer: results from a referral center for early esophageal cancer. Endoscopy 2010; 42: $456-461$

[15] Zhang JQ, Hooker CM, Brock MV et al. Neoadjuvant chemoradiation therapy is beneficial for clinical stage T2 N0 esophageal cancer patients due to inaccurate preoperative staging. Ann Thorac Surg 2012; 93: 429-435; discussion 436-427

[16] The Paris endoscopic classification of superficial neoplastic lesions: esophagus, stomach, and colon: November 30 to December 1, 2002. Gastrointest Endosc; 2003; 58: S3 - S43

[17] Shaheen NJ, Falk GW, Iyer PG et al. ACG Clinical Guideline: Diagnosis and management of Barrett's esophagus. Am | Gastroenterol 2016; 111: 30 - 50, quiz 51
[18] Fotis D, Doukas M, Wijnhoven BPL et al. Submucosal invasion and risk of lymph node invasion in early Barrett's cancer; potential impact of different classification systems on patient management. United European Gastroenterol J 2015; 3: 505- 513

[19] Boys JA, Worrell SG, Chandrasoma P et al. Can the risk of lymph node metastases be gauged in endoscopically resected submucosal esophageal adenocarcinomas? A multi-center study J Gastrointest Surg 2016; 20: 6-12; discussion 12

[20] Manner $\mathrm{H}$, Pech O, Heldmann $\mathrm{Y}$ et al. The frequency of lymph node metastasis in early-stage adenocarcinoma of the esophagus with incipient submucosal invasion (pT1b sm1) depending on histological risk patterns. Surg Endosc 2015; 29: $1888-1896$

[21] Sepesi B, Watson TJ, Zhou D et al. Are endoscopic therapies appropriate for superficial submucosal esophageal adenocarcinoma? An analysis of esophagectomy specimens ] Am Coll Surg 2010; 210: 418 427

[22] Alvarez Herrero L, Pouw RE, van Vilsteren FG et al. Risk of lymph node metastasis associated with deeper invasion by early adenocarcinoma of the esophagus and cardia: study based on endoscopic resection specimens. Endoscopy 2010; 42: 1030-1036

[23] Choi J, Kim SG, Kim JS et al. Comparison of endoscopic ultrasonography (EUS), positron emission tomography (PET), and computed tomography $(\mathrm{CT})$ in the preoperative locoregional staging of resectable esophageal cancer. Surg Endosc 2010; 24: 1380 - 1386

[24] Puli SR, Reddy JB, Bechtold ML et al. Staging accuracy of esophageal cancer by endoscopic ultrasound: a meta-analysis and systematic review. World J Gastroenterol 2008; 14: 1479-1490

[25] Rice TW, Mason DP, Murthy SC et al. T2NOM0 esophageal cancer. ] Thorac Cardiovasc Surg 2007; 133: 317 - 324

[26] Lee L, Ronellenfitsch U, Hofstetter WL et al. Predicting lymph node metastases in early esophageal adenocarcinoma using a simple scoring system. J Am Coll Surg 2013; 217: 191 - 199

[27] Leers JM, DeMeester SR, Oezcelik A et al. The prevalence of lymph node metastases in patients with T1 esophageal adenocarcinoma a retrospective review of esophagectomy specimens. Ann Surg 2011; 253: $271-278$

[28] Alvarez Herrero L, Pouw RE, van Vilsteren FG et al. Safety and efficacy of multiband mucosectomy in 1060 resections in Barrett's esophagus. Endoscopy 2011; 43: $177-183$

[29] Pech O, May A, Manner $\mathrm{H}$ et al. Long-term efficacy and safety of endoscopic resection for patients with mucosal adenocarcinoma of the esophagus. Gastroenterology 2014; 146: 652-660 .e651

[30] Pouw RE, Peters FP, Sempoux C et al. Stepwise radical endoscopic resection for Barrett's esophagus with early neoplasia: report on a Brussels' cohort. Endoscopy 2008; 40: 892-898

[31] Terheggen G, Horn EM, Vieth M et al. A randomised trial of endoscopic submucosal dissection versus endoscopic mucosal resection for early Barrett's neoplasia. Gut 2017; 66: $783-793$

[32] Komeda $\mathrm{Y}$, Bruno M, Koch A. EMR is not inferior to ESD for early Barrett's and EG] neoplasia: An extensive review on outcome, recurrence and complication rates. Endosc Int Open 2014; 2: E58 - E64

[33] Metzger R, Bollschweiler E, Vallbohmer D et al. High volume centers for esophagectomy: what is the number needed to achieve low postoperative mortality? Dis Esophagus 2004; 17: 310 - 314

[34] May A, Gunter E, Roth F et al. Accuracy of staging in early oesophageal cancer using high resolution endoscopy and high resolution endosonography: a comparative, prospective, and blinded trial. Gut 2004; 53: $634-640$

[35] Pouw RE, Heldoorn N, Alvarez Herrero L et al. Do we still need EUS in the workup of patients with early esophageal neoplasia? A retrospective analysis of 131 cases Gastrointest Endosc 2011; 73: 662 - 668 